\title{
Merkblatt zum Antragsverfahren auf Modifikation, Ergänzung, Streichung von Leistungen der TARMED-Tarifstruktur im Rahmen der ordentlichen Tarifpflege
}

\section{Wer ist antragsberechtigt?}

1. Antragsberechtigt sind Personen, die von Fachgesellschaften gemeldet werden, die in der Ärztekammer stimmberechtigt sind und/ oder in der KWFB einen Fähigkeits- oder Fertigkeitsausweis vertreten.

2. Pro Fachgesellschaft darf nur eine (1) Person gemeldet werden.

3. Die gemeldete Person muss Internetzugang über HIN/ASAS haben.

4. Mitglieder der TAKO (Tarifkommission FMH) sind ebenfalls antragsberechtigt.

\section{Wie erfolgt die Meldung der berechtigten Person?}

5. Die Meldung der berechtigten Person erfolgt durch den Präsidenten der entsprechenden Fachgesellschaft.
Abbildung 1

Eingabemaske.
6. Sie bedarf der Schriftform und muss entweder per regulärer Post oder auf dem elektronischen Weg (E-Mail) an den FMH-Tarifdienst gerichtet werden.

\section{Was sind die technischen Voraus- setzungen für die Antragstellung?}

7. Die Antragstellung erfolgt elektronisch mittels einer eigens dafür eingerichteten Antragsdatenbank.

8. Die Datenbank wird im Internet unter www.fmh-re2.ch gehostet und ist den Berechtigten zugänglich.

9. Die gemeldete Person erhält nach der Überprüfung der Antragsberechtigung (Fachgesellschaft, Antragsteller, Internetzugang) die notwendigen Informationen für die Benutzung des Antragsprogramms. Sie erhält ebenfalls Instruktionen/Hinweise zu dessen Benutzung.

\section{Abbildung 2}

Helpfile.

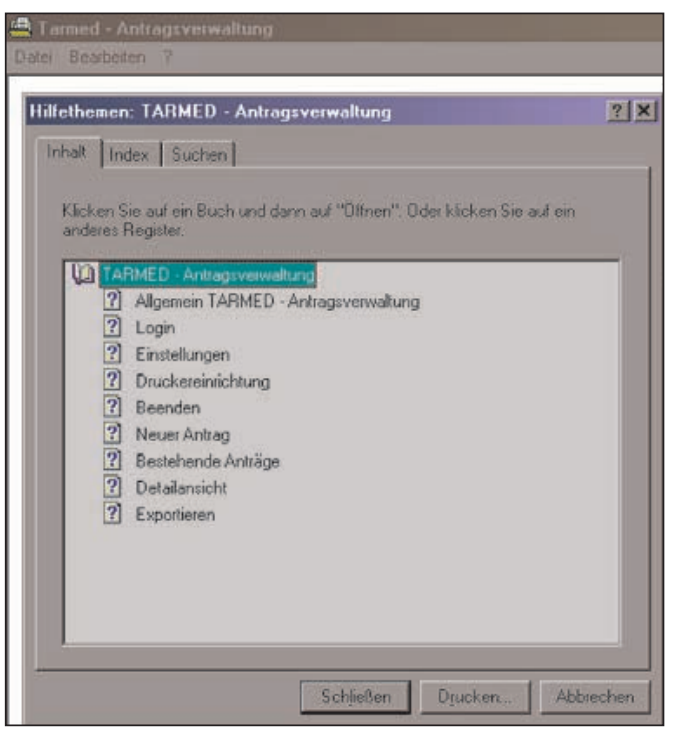

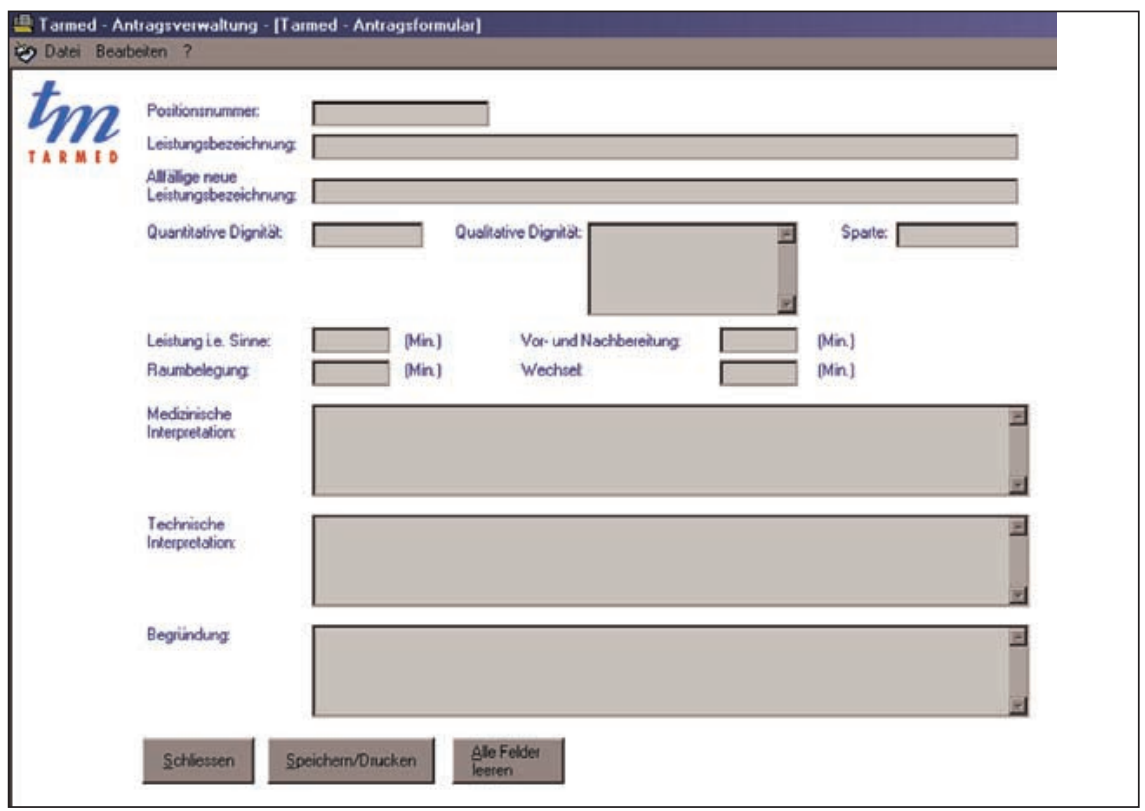


Tabelle 1

Grobübersicht Zeitplan.

\begin{tabular}{|c|c|c|c|c|c|c|c|c|}
\hline 1.1. & $t-1$ & 31.12 . & 1.1. & to & 31.12. & 1.1. & $t+1$ & 31.12. \\
\hline \multicolumn{3}{|c|}{$\rightarrow$ Antrag auf Modifikation } & \multicolumn{3}{|c|}{$\begin{array}{l}\text { Bearbeitung, Entscheid } \\
\text { (inkl. TPW-Verhandlung) }\end{array}$} & \multicolumn{3}{|c|}{ Inkraftsetzung $\rightarrow$} \\
\hline
\end{tabular}

10. Jeder berechtigte Antragsteller erhält vom Tarifdienst sein Login/Passwort. Mit diesem kann er sich Zugang zum Antragsformular beschaffen. Es können nur die eigenen Anträge eingesehen werden. Eine Veränderung der Datenbank selbst ist ausgeschlossen.

11. Die Datenbank kann sowohl mit Access als auch unter Excel durch die für die Bearbeitung der Anträge zuständigen Personen bearbeitet werden.

12. Mit diesen Mitteln können die Anträge erfasst werden.

\section{Wie läuft das Antragsverfahren ab?}

13. Die Anträge werden per Mail direkt in die Antragsdatenbank transferiert. Der Antragsteller sieht anhand seiner Mailliste, welche Anträge er gestellt hat.

14. Jeder Antrag muss im Prinzip auf eine Einzelposition des Tarifes bezogen sein. Die zu verändernden Eckwerte müssen angegeben sein. Jeder Antrag, jede Veränderung ist im entsprechenden Feld zu begründen.

15. Für neue Tarifpositionen kann eine Dummy-Nummer eingegeben werden. Im Rahmen der Tarifpflege wird bei einem positiven Entscheid die definitive Positionsnummer zugeteilt.
16. Jeder Antrag ist gekennzeichnet mit dem Login des Antragstellers. So kann jeder Antrag einer Person zugeordnet werden.

17. Der Antragsteller erhält eine schriftliche Bestätigung über die von ihm eingereichten Anträge. Der Entscheid des für die Tarifpflege zuständigen Organs wird jeweils im April des Folgejahres mitgeteilt.

\section{Welche Fristen gilt es zu berücksichtigen?}

18. Die Tarifrevisionen finden einmal jährlich statt.

19. Anträge, die im Jahr «t $+1 »$ wirksam werden sollen, müssen per 31. Dezember des Jahres « $\mathrm{t}-1$ » eingereicht sein (siehe Tabelle 1).

20. Damit die beantragten Änderungen der Abrechnung zugeführt werden können, sind im KVG-Bereich (welcher für die Terminplanung massgeblich ist) folgende Schritte zu berücksichtigen (siehe Tabelle 2 ).

\section{Ab wann können erstmals Anträge eingereicht werden?}

21. Die Anträge auf Tarifrevision können beginnend per 1. Juli 2002 eingereicht werden.

22. Die Bearbeitung dieser Anträge findet $a b$ erstem Quartal 2003 statt.

23. Die revidierten Positionen finden in der ersten ordentlichen Revision Eingang, die auf den 1. Januar 2004 wirksam wird.

Tabelle 2

Detailübersicht Zeitplan (Jahr «t0»).

\begin{tabular}{|c|c|c|c|c|c|c|c|}
\hline 31. 3 & 1.4 . & 30.6 & 1.7. & 30.9. & 1.10 & 31.12 . & \\
\hline $\begin{array}{l}\text { Bearbeitung der Anträge } \\
\text { im Rahmen der NFO; } \\
\text { inkl. Gespräche mit den } \\
\text { betroffenen FG } \\
\text { (falls erforderlich) }\end{array}$ & \multicolumn{2}{|c|}{$\begin{array}{c}\text { (summarisches) } \\
\text { Genehmigungsverfahren } \\
\text { Bundesrat; } \\
\text { TPW-Verhandlungen } \\
\text { in den Kantonen }\end{array}$} & \multicolumn{2}{|c|}{$\begin{array}{l}\text { TPW-Genehmigungs- } \\
\text { verfahren in den Kantonen; } \\
\text { inkl. Anhörung } \\
\text { Preisüberwachung }\end{array}$} & \multicolumn{2}{|c|}{$\begin{array}{l}\text { Implementierung neue } \\
\text { Tarifstruktur/TPW } \\
\text { bei Leistungserbringern } \\
\text { und Versicherern }\end{array}$} & \\
\hline \multicolumn{2}{|c|}{$\begin{array}{c}\text { Vorliegen revidierte } \\
\text { Tarifstruktur }\end{array}$} & $\begin{array}{r}\text { Vorlieg } \\
\text { Tarifs } \\
\text { ausge }\end{array}$ & $\begin{array}{l}\text { hmigte } \\
\text { sowie } \\
\text { TPW }\end{array}$ & \multicolumn{2}{|c|}{$\begin{array}{c}\text { Vorliegen genehmigte } \\
\text { TPW }\end{array}$} & \multicolumn{2}{|c|}{$\begin{array}{l}\text { Vorliegen sämtlicher } \\
\text { Voraussetzungen } \\
\text { für die Abrechnung }\end{array}$} \\
\hline
\end{tabular}

